\title{
Light extraction from GaN-Microcavity
}

\author{
Naser M. Ahmed, Z. Hassan \\ Nano-Optoelectronics Research and Technology Laboratory, \\ School of Physics, University Science Malaysia, 11800 Penang, Malaysia \\ *E-mail of Corresponding author: naser@usm.my
}

Keywords: GaNMicrocavity, UV light enhancement, critical angle.

\begin{abstract}
It will be demonstrated that light extraction of GaNcan be more efficient with the use of flat and dome epoxy. Theoretically the angle, wavelength, and thickness depend on the photoluminescence emission of the luminescent film of transparent substrate. This was studiedusing theFresnel type transmittance calculations for s- (TE) and p- (TM) polarized emission. Experimentally we have demonstrated a GaN/sapphire microcavity exhibited in the 1.3-1.6 fold enhancement in light extraction efficiency by using flat and dome epoxy as external medium compared with air external medium. In addition, simulation results shows that using $\left(\mathrm{HfO}_{2}\left(\theta_{c}=52.7^{0}\right)\right.$, Epoxy $\left(\theta_{c}=36.8^{0}\right), \mathrm{MgF}_{2}\left(\theta_{c}=33.7^{0}\right)$, Air $\left.\left(\theta_{c}=23.5^{0}\right)\right)$ improve the light extraction by increasingthe critical angle and diffracting the internal light with a large solid angle into the light escape cone.
\end{abstract}

\section{Introduction}

Light emitting diodes (LEDs) have undergone a huge improvement from the weak lights they used to be twenty years ago to the ultimate lamps they have the potential to become tomorrow [1]. A new generation of devices, "high-brightness" LEDs, now account for the largest poration of the LED market. This evolution is the consequence not only from the emergence of nitride semiconductor compounds completing the rainbow of emitted colors, but also from the optical design of LED. For a typical III-V compound such as GaN only the light emitted in directions close to the normal to the top surface escapes the LED (Fig. 1). Thus, the efficiency of "conventional" LEDs is limited to 
$\left(1 / 4 n^{2}\right) \sim 4 \%$ in air ( $8 \%$ in epoxy) [2]. The remainder of internally generated light $(92 \%-96 \%)$ never has an opportunity to escape the emitter, and is eventually absorbed, either by the active region or the opaque substrate. For years, a significant amount of scientific work has been focused on ways of improving the extraction efficiency of the light-emitting diode (LED). Many interesting approaches have been proposed to accomplish this, such as the use of thin light emitting layers with surface texturing [3], resonant cavities [4], photon recycling [5], or output coupling through surface plasmons excited at metal surface [6]. In this paper, we present a theoretical analysis of the microcavity structure using GaN asan emitter thin film and sapphire substrate, the angle dependent transmission is determined theoretically by applying the Fresnel equations of reflection and transmission. In addition, using photoluminescence measurements we compared the theoretical results with experimental results using epoxy as an external medium.

\section{Microcavity Principle of Operation}

The model of light emission from $\mathrm{GaN}$ is based on the diffraction scattering of light from the various interfaces (air/film: interface, film/substrate: interface) shown in Fig.1. Efficient planar LEDs are not easy to make,the main difficulty comes from the large refractive index difference between the semiconductor $\left(\boldsymbol{n}_{f}\right)$ and the outside medium $\left(\boldsymbol{n}_{\text {ext }}\right)$. According to the Snell- Descartes law, light propagating inside the semiconductor can only be extracted if it fits within the cone defined by the critical angle of total internal reflection $\theta_{\mathrm{c}}=\arcsin \left(\frac{n_{\text {ext }}}{n_{f}}\right)$ with the normal of the interface. The rest of the light is totally internally reflected back into the semiconductor. For a GaN LED $n_{f}=2.5$ and the critical angle is about $23.5^{\circ}$. The surface optical extraction factor $\left(\eta_{\text {ext }}\right)$ is simply given by a ratio of solid angles multiplied by the Fresnel transmission factor $\left(T_{\text {Fresnel }}\right)$ [7].

$\eta_{\text {ext }} \approx \frac{2 \pi\left(1-\cos \theta_{c}\right)}{4 \pi} T_{\text {Fresnel }} \approx \frac{n_{\text {ext }}^{2}}{4 n_{f}^{2}} T_{\text {Fresnel }}$ 
Eq. 1 giving $2.4 \%$ for an extraction into air, and 5.4\% into epoxy. Only the photons emitted into this escape cone will be extracted, the rest are bounced back at the interface due to the total internal reflection (TIR). The extraction efficiency is given by the fraction of the photons emitted in the escape cone (assuming an isotropic power distribution inside the semiconductor $\mathrm{P}(\theta)$ ) [8]:

$\eta_{\text {ext }}=\frac{\int_{0}^{\arcsin } P(\theta) 2 \pi \sin (\theta) d \theta}{\int_{0}^{\frac{1}{n}} P(\theta) 2 \pi \sin (\theta) d \theta} \approx \frac{1}{4 n^{2}}$

The extremely low extraction efficiency limits the employment of LEDs in many applications. Several solutions to increase the extraction efficiency have been proposed. Embedding the LED in a transparent epoxy dome with a high refractive index can increase the critical angle. The light can be extracted from multiple output planes [9]. Other techniques alter the path of the photon until it hits the semiconductor air interface within the extraction cone. Using this approach, in combination with multiple-plane extraction, world-record efficiencies up to $55 \%$ at room temperature $(32 \%$ without the epoxy dome) have been obtained [10]. Overall efficiencies up to $41 \%$ have been achieved, and up to $55 \%$ is achieved when the (Non-resonant cavity LED) is covered with an epoxy dome [11]. The active region can reabsorb the photons, which undergo the total internal reflection, and the generated carriers can afterwards recombine again, increasing the chance that the new photon is emitted into the good solid angle. This effect has been known for a long time, and has been used in LEDs to increase efficiency [12]. The angular power distribution inside the device can be altered, in such a way that more light is emitted in the extraction cone. 


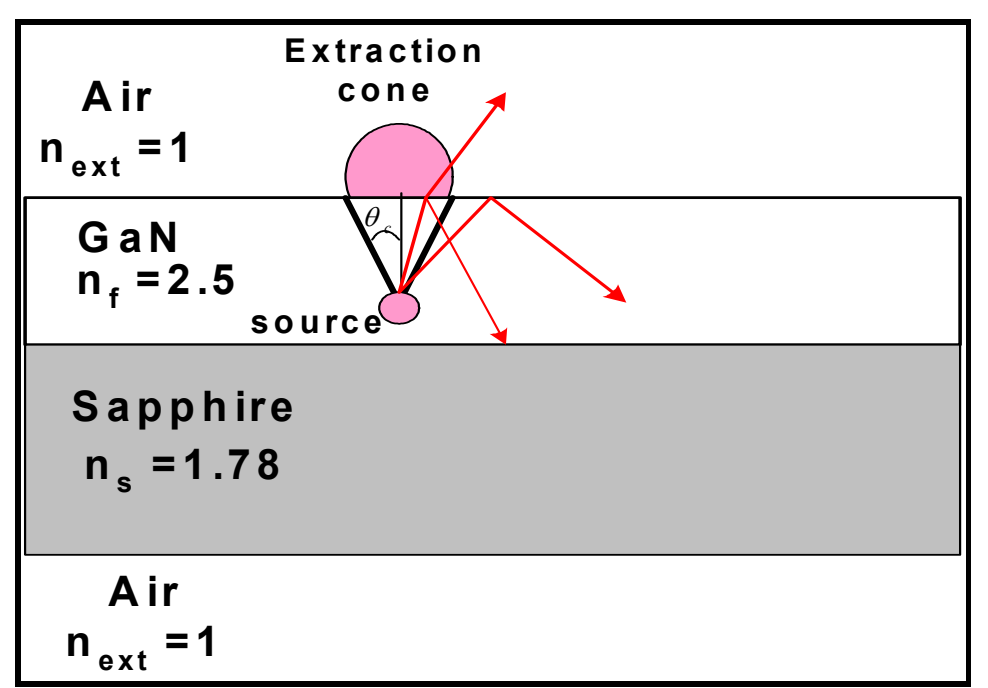

Fig. 1. GaN cavity structure.

\section{Microcavity Analysis}

The light emission from the thin emitting film on a substrate is studied. The emission of light to the airside and to the substrate side is considered. The airside thickness and the substrate thickness are unlimited. A schematic drawing is shown in Fig. 2(a). S-polarized light (TE mode propagation, electric field vector parallel to film plane) and p-polarized light (TM mode propagation, electric field vector in plane of propagation perpendicular to film plane) are considered. The situation of reflection and refraction at an interface, $J$, with refractive indices $n_{f}>n_{s}$ is illustrated in Fig. 2(b). The amplitude coefficients of reflection, $r_{i j}$, and transmission, $t_{i j}$, of the electrical field strength at an angle of incidence, $\phi_{i}$, are less than the angle of total internal reflection, $\phi_{i, t}$, and are at an angle of refraction, $\phi_{j}$, as demonstrated by [13].

$r_{i j}^{s}=\frac{n_{i} \cos \left(\phi_{i}\right)-n_{j} \cos \left(\phi_{j}\right)}{n_{i} \cos \left(\phi_{i}\right)+n_{j} \cos \left(\phi_{j}\right)}$

$r_{i j}^{p}=\frac{n_{j} \cos \left(\phi_{i}\right)-n_{i} \cos \left(\phi_{j}\right)}{n_{j} \cos \left(\phi_{i}\right)+n_{i} \cos \left(\phi_{j}\right)}$ 
$t_{i j}^{s}=\frac{2 n_{i} \cos \left(\phi_{i}\right)}{n_{i} \cos \left(\phi_{i}\right)+n_{j} \cos \left(\phi_{j}\right)}$

$t_{i j}^{p}=\frac{2 n_{i} \cos \left(\phi_{i}\right)}{n_{j} \cos \left(\phi_{i}\right)+n_{i} \cos \left(\phi_{j}\right)}$

whereby

$\phi_{i}=\arcsin \left(\frac{n_{j}}{n_{i}} \sin \left(\phi_{j}\right)\right)$

The superscripts $\mathrm{s}$ and $\mathrm{p}$ stand for $\mathrm{s}-$ and $\mathrm{p}$ polarized light, respectively. For $n_{i}>n_{j}$ the angle of total internal reflection, $\phi_{i, t}$, is

$\phi_{i, t}=\arcsin \left(\frac{n_{j}}{n_{i}}\right)$

For parallel-polarised light the Brewster angle, $\phi_{j, B}$, is

$\phi_{j, B}=\arctan \left(\frac{n_{i}}{n_{j}}\right)$

In the case of $n_{i}>n_{j}$ and $\phi_{i}>\phi_{i, t}$ the electric field amplitude transmission coefficients are

$t_{i j}^{s}=t_{i j}^{p}=0$

and the amplitude reflection coefficients are [13]

$r_{i j}^{\mu}=\exp \left(2 i \phi_{i j}^{\mu}\right), \quad \mu=s, p$

with the phase shifts 
$\phi_{i j}^{s}=\arctan \left[\frac{\left[n_{i}{ }^{2} \sin ^{2}\left(\phi_{i}\right)-n_{j}^{2}\right]^{1 / 2}}{n_{i} \cos \left(\phi_{i}\right)}\right]$

and

$\phi_{i j}^{p}=\arctan \left[\frac{n_{i}\left[n_{i}{ }^{2} \sin ^{2}\left(\phi_{i}\right)-n_{j}{ }^{2}\right]^{1 / 2}}{n_{j}{ }^{2} \cos \left(\phi_{i}\right)}\right]$

The phase change between equal wave fronts of a directly refracted ray at interface, $\mathrm{J}$, and a ray refracted at $\mathrm{J}$ after reflections at $\mathrm{J}$ and I (see Fig. 1(b)) is given by

$\phi_{\text {path }}=2 x_{1} k_{i}-x_{3} k_{j}$

where

$x_{1}=\frac{d_{f}}{\cos \left(\phi_{i}\right)}$

$x_{2}=d_{f} \tan \left(\phi_{i}\right)$

$x_{3}=2 x_{2} \sin \left(\phi_{j}\right)=2 d_{f} \tan \left(\phi_{i}\right) \sin \left(\phi_{j}\right)$

$k_{\mu}=n_{\mu} 2 \pi / \lambda, \quad \mu=i, j$

$k_{\mu}$ is the wave vector in medium $\mu$, and $\lambda$ is the wavelength in vacuum. The total phase difference between a beam being reflected once at both interfaces, $\mathrm{J}$ and $\mathrm{I}$, before transmitted at $\mathrm{J}$ and a beam directly transmitted at interface $\mathrm{J}$ is 
$\Delta \phi_{J}^{\mu}=\phi_{J I J}^{\mu}-\phi_{J}^{\mu}=\phi_{i j}^{\mu}+\phi_{i h}^{\mu}+\phi_{p a t h}$

Constructive interference occurs if

$\Delta \phi_{J}^{\mu}=2 \pi m \quad m=0,1,2,3, \ldots \ldots$

while destructive interference occurs if

$\Delta \phi_{J}^{\mu}=2 \pi(m+1 / 2) \quad m=0,1,2,3, \ldots \ldots$

$m$ is the interference order.

The total electric field transmission, $t_{i j}^{\mu}$, at interface $J$ is obtained by the geometric row of the direct transmission and the transmissions after multiple reflection according to

$t_{i j, J}^{\mu}=\frac{t_{i j}^{\mu}}{1-\exp \left(i \phi_{p a t h}\right) r_{i j}^{\mu} r_{i h}^{\mu}}$

The intensity transmission from $\mathrm{i}$ to $\mathrm{j}$ at interface $\mathrm{J}$ is given by [13].

$T_{i j, J}^{\mu}=\frac{I_{j}}{I_{i}}=\frac{n_{j} \cos \left(\phi_{j}\right)}{n_{i} \cos \left(\phi_{i}\right)}\left|t_{i j, J}^{\mu}\right|^{2}$ 

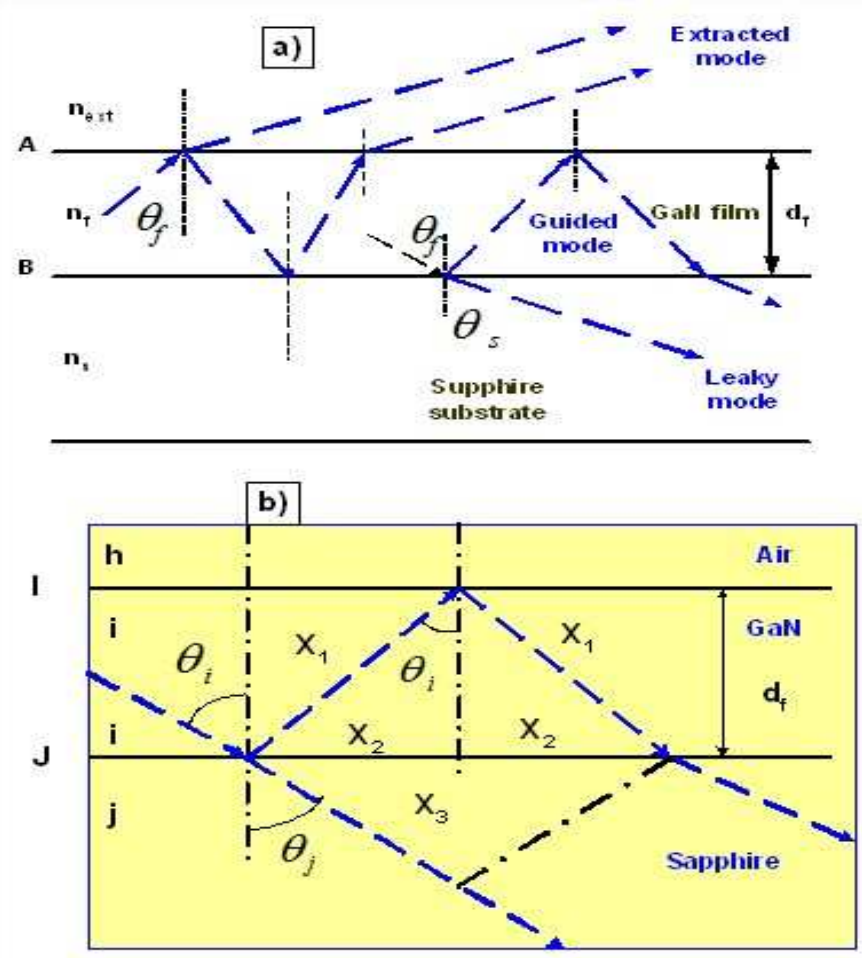

Fig. 2. (a) Schematic of ray path through thin film on substrate caused by light emission in film.

Transmissions at interface A to air and at interface B to the substrate are indicated. (b) Detailed drawing of ray path by direct transmission at interface $\mathrm{J}$ and after reflection at $\mathrm{J}$ and $\mathrm{I}$.

\section{Simulation and experimental results}

In the simulations a luminescent film on a transparent substrate is considered. There is transmission from the film to the air at the film-air interface, A, and the transmission from the film to the substrate at the film-substrate interface, $\mathrm{B}$, is simulated. The refractive indices are fixed to $\mathrm{n}_{\mathrm{ext}}=1$ for the air, $\mathrm{n}_{\mathrm{f}}=2.5$ for the $\mathrm{GaN}$ film, and $\mathrm{n}_{\mathrm{s}}=1.78$ for the sapphire substrate. The angles of transmission, $\Phi_{\mathrm{a}}$ (at air side) and $\Phi_{\mathrm{s}}$ (at substrate side), the film thickness, $\mathrm{d}_{\mathrm{f}}$, is varied. In Fig. 3 the intensity reflectance at the film-air interface, $\mathrm{R}_{\mathrm{A} ; \mathrm{s}}=\left|\boldsymbol{r}_{i h}^{s}\right|^{2}$ of s-polarised light, $\mathrm{R}_{\mathrm{A} ; \mathrm{p}}=\left|\boldsymbol{r}_{i h}^{p}\right|^{2}$ of p-polarised light, and the intensity reflectance at the film-substrate interface, $\mathrm{R}_{\mathrm{B} ; \mathrm{s}}=\left|\boldsymbol{r}_{i j}^{s}\right|^{2}$ of s-polarised light, $\mathrm{R}_{\mathrm{B} ; \mathrm{p}}=$ 
$\left|r_{i j}^{p}\right|^{2}$ of p-polarised light is displayed. The zero reflectance of the Brewster angle for p-polarised light, and the rise in reflectance towards 1 near the critical angle of total internal reflection are clearly seen.

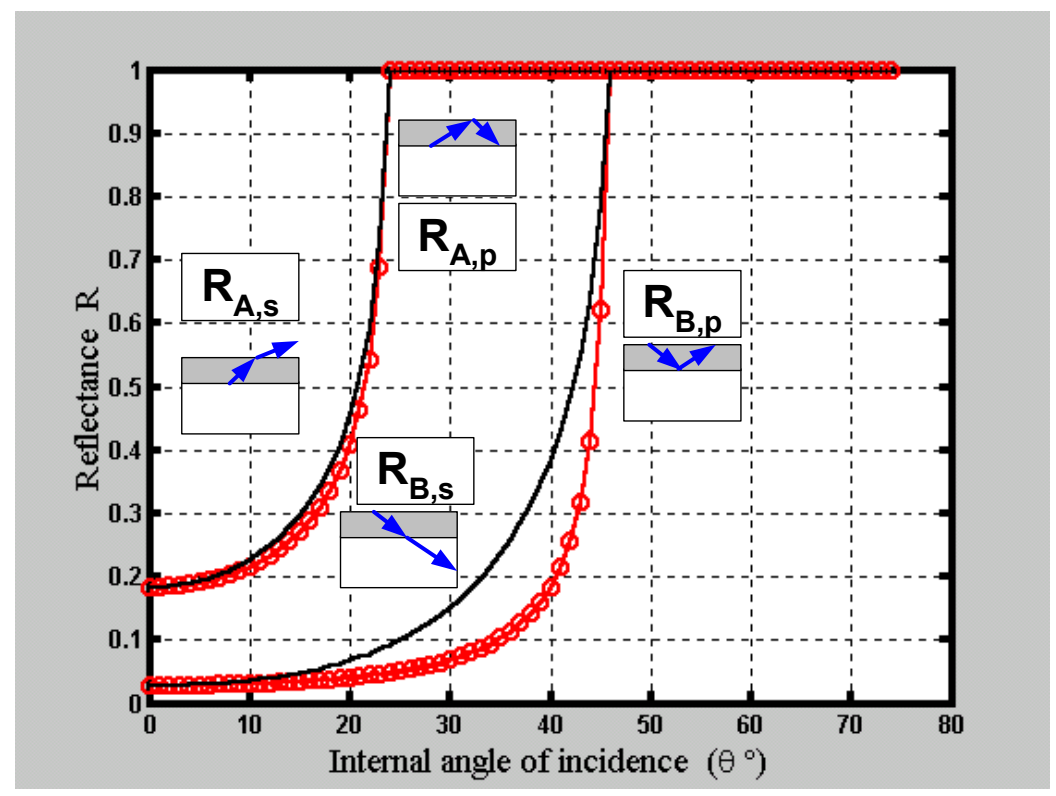

Fig. 3. Single-interface intensity reflectance versus angle of incidence for propagation from optical denser to optical thinner medium. Situations are presented for s- and p-polarised light at film-air interface, A, and at film-substrate interface, B. Applied refractive indices are $\mathrm{n}_{\mathrm{ext}}=1, \mathrm{n}_{\mathrm{f}}=2.5$, and

$$
\mathrm{n}_{\mathrm{s}}=1.78 \text {. }
$$

Similarly, in Fig. 4 we can see the increasing of the critical angle simulation results by embedding $\mathrm{GaN}$ in a transparent epoxy $(\mathrm{n}=1.5), \mathrm{MgF}_{2}(\mathrm{n}=1.39)$, and $\mathrm{HfO}_{2}(\mathrm{n}=1.99)$. This implies that move light escapes from the $\mathrm{GaN}$ in to these materials. 


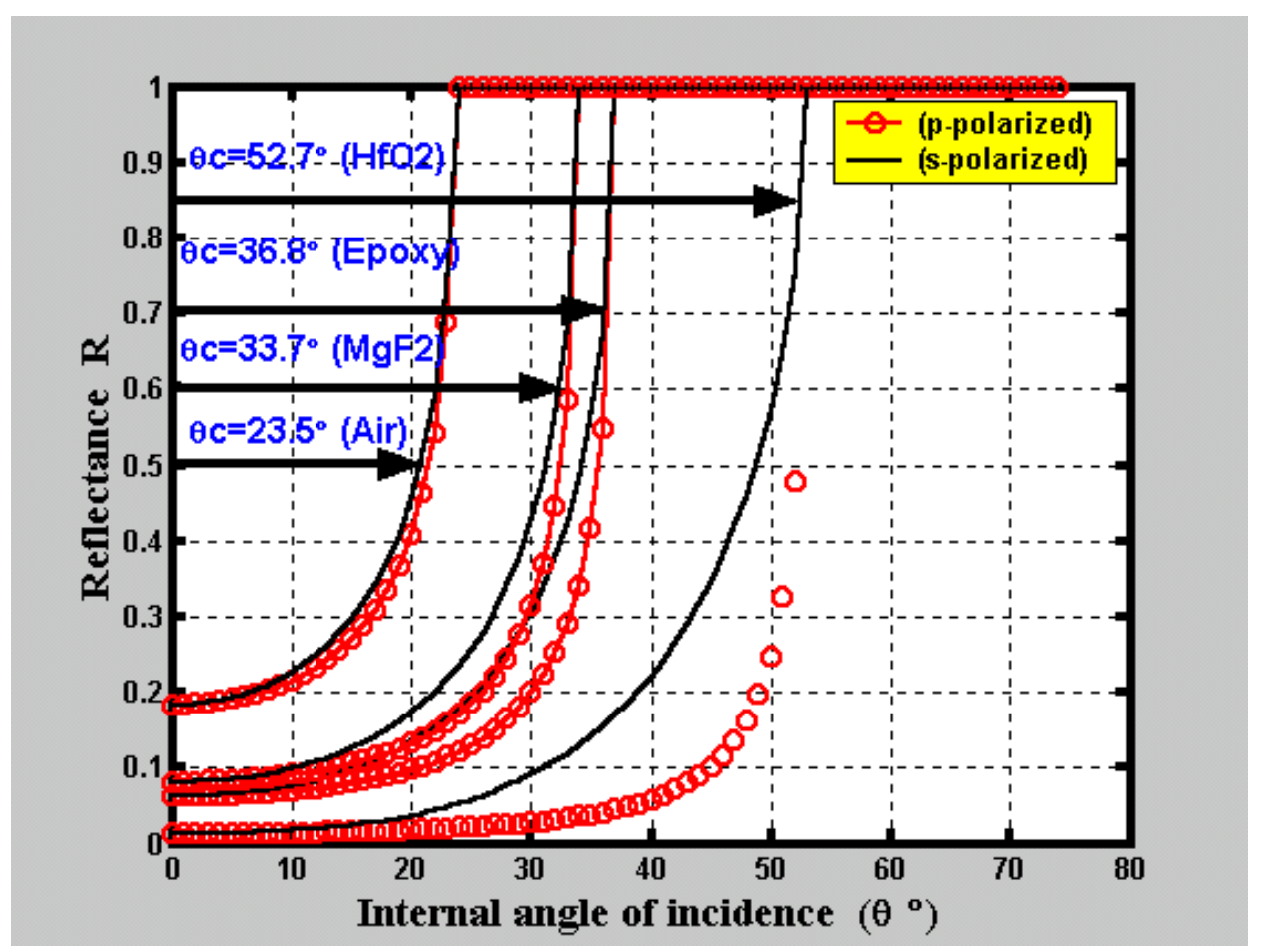

Fig. 4. Single-interface intensity reflectance versus internal angle of incidence using different material as external medium $\left(\mathrm{HfO}_{2}\right.$, epoxy, $\mathrm{MgF}_{2}$, air).

Fig. 5 shows the PL spectrum for the three cavities (GaN/sapphire, flat epoxy/GaN/sapphire and dome epoxy/GaN/sapphire) to change the external medium refractive index and enhance the photoluminescence. Clearly the PL intensity of the flat epoxy cavity experience is more than 1.3-fold enhancement while the dome epoxy cavity experience is more than 1.6-fold enhancement compared to the cavity without epoxy. 


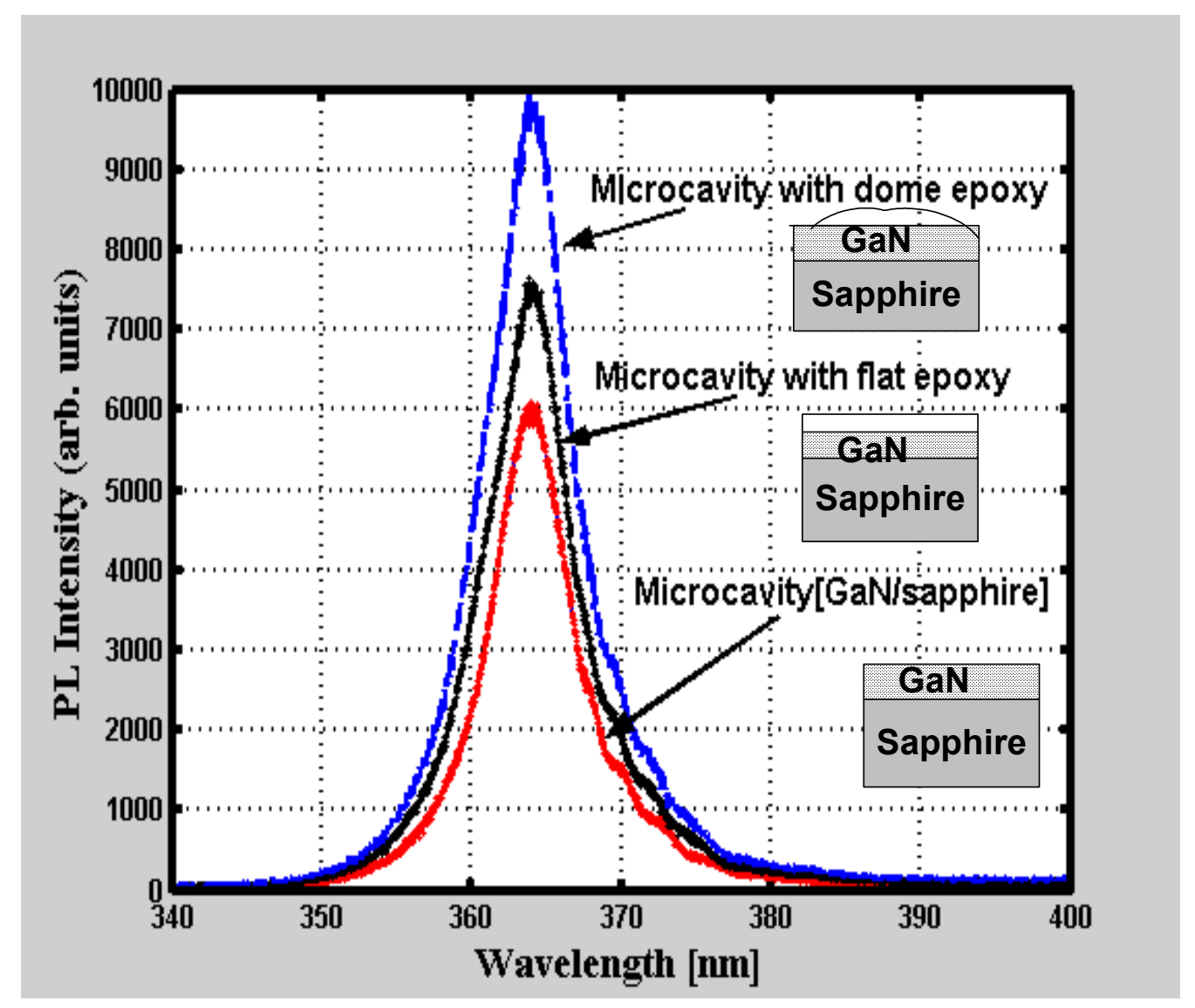

Fig. 5. PL enhancement spectra for GaNmicrocavity using flat and dome epoxy.

In Fig. 6 the emission behaviors from film to air and from film to substrate at normal incidenceare illustrated. In Fig. 6 the film thickness is varied at constant wavelength, while there are $2 \pi$ phase differences between s- (TE) and p- (TM) polarised light, which gives no difference in the transmission of s- and p-polarised light. The transmission modulates with film thickness (Fig. 6(a)) due to the constructive and destructive multi-beam interference at the interfaces. The transmission at the film- air interface is lower than the film-substrate interface because there is a larger refractive index step at the film-air border than at the film-substrate border. 


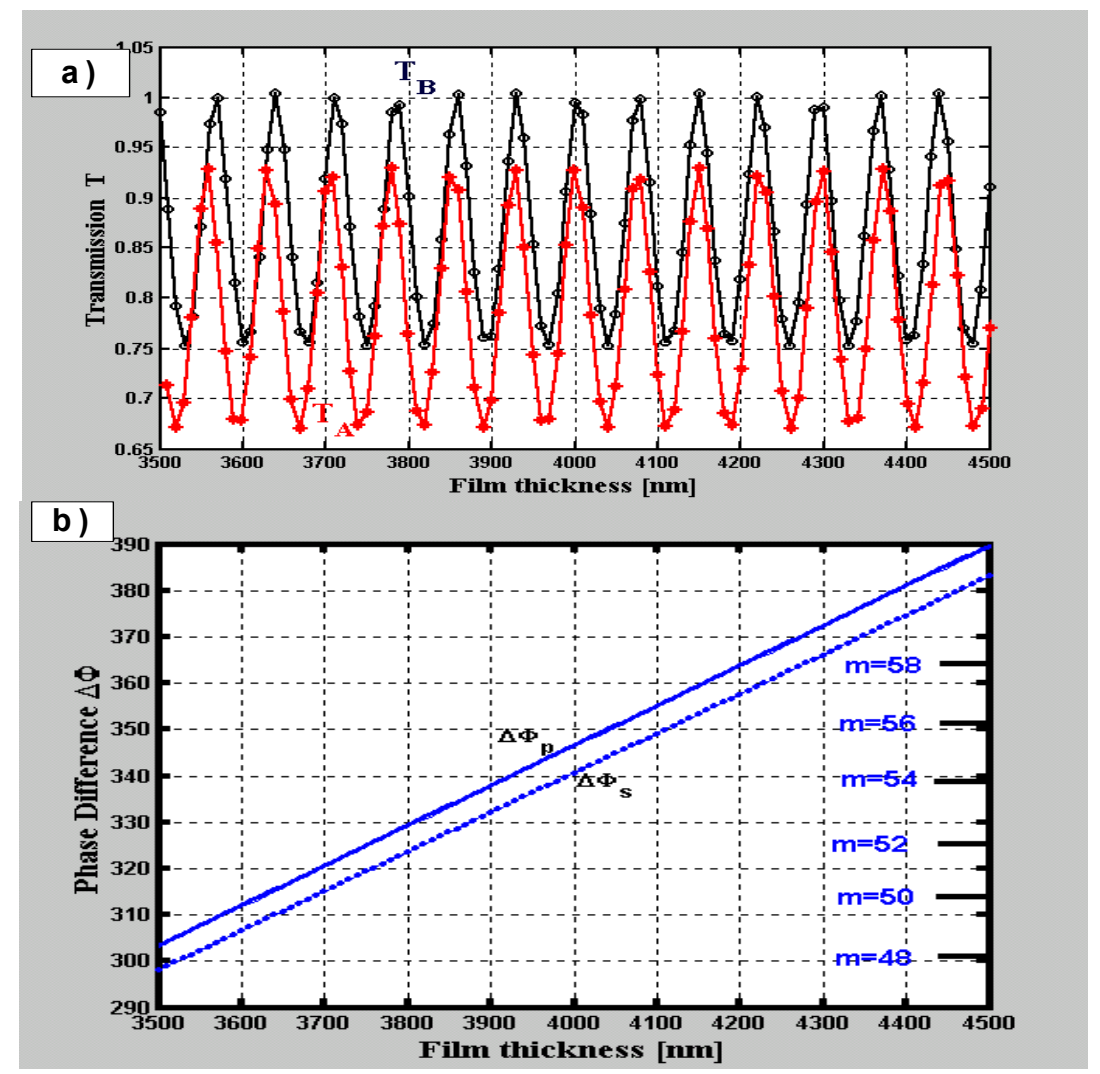

Fig. 6. Simulation of thickness dependence of transmission at film-air interface, TA, and at filmsubstrate interface, $\mathrm{TB}$, in the case of normal incidence, $\theta_{f}=0$. Used parameters: Wavelength $\lambda=364 \mathrm{~nm}$; refractive indices $n_{\text {ext }}=1, n_{f}=2.5, n_{s}=1.78$. (a) Transmissions (no difference between s- and p-polarised lights). (b) Phase differences, $\Delta \phi_{s}$ and $\Delta \phi_{p}$, between double reflected beam before transmission and directly transmitted beam (Eq. (18), no differences at interfaces A and B). The interference order $\mathrm{m}$ is indicated.

In Fig.7 shows the simulation results of the transmission light from microcavity when we using epoxy compared with cavity without epoxy, clearly we can see that the epoxy enhance the light transmission from the cavity by 1.1 -fold at normal incidence $\left(\theta_{f}=0\right)$. 


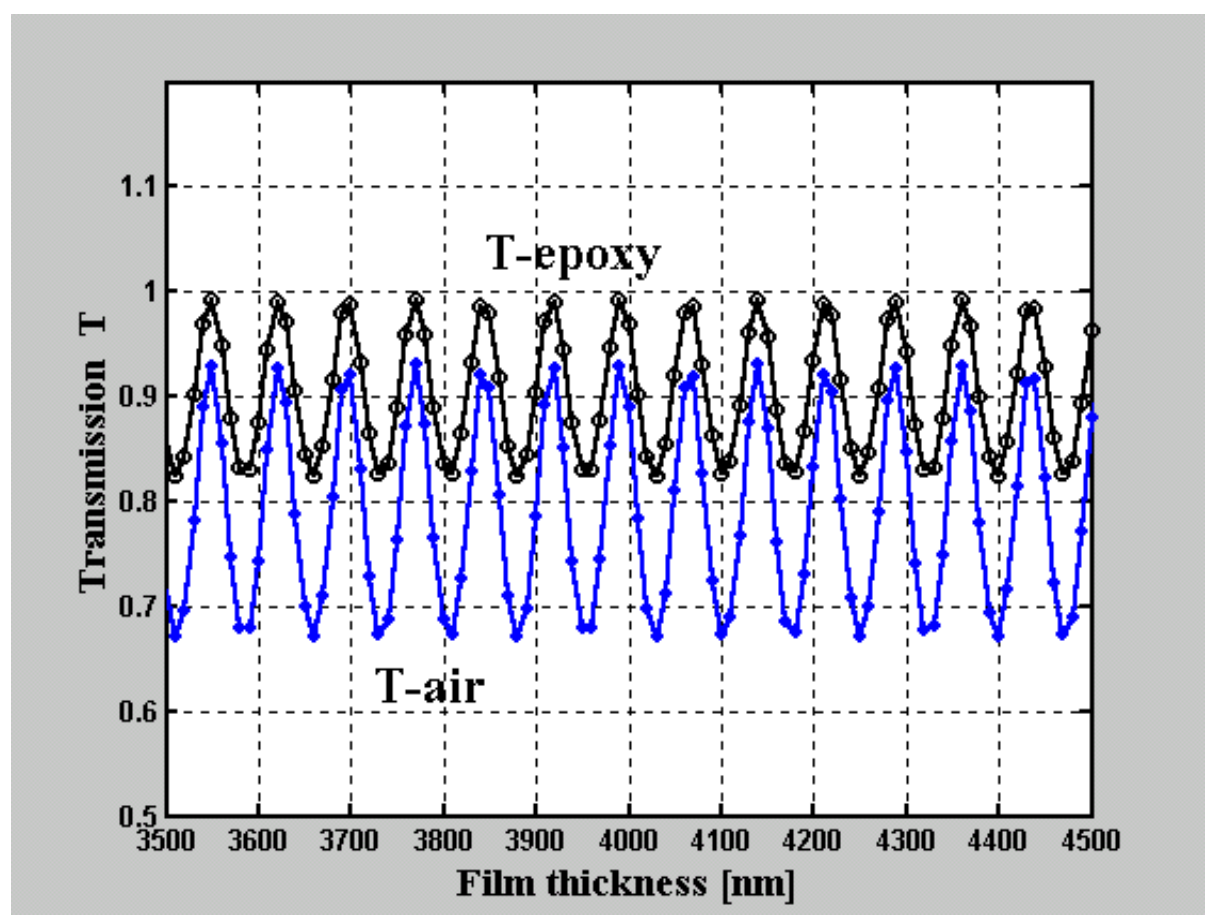

Fig. 7. Simulation of thickness dependence of transmission at film-air interfaces and film-epoxy interfaces at ( $\theta_{f}=0, \lambda=364 \mathrm{~nm}, n_{f}=2.5, n_{\text {epoxy }}=1.5$ ).

\section{Conclusions}

The light transmission of luminescent films on transparent substrates of lower refractive index than the films was studied theoretically. The basic physics of the microcavity was described. Some microcavity designs were given, to improve the light extraction and enhance the spontaneous emission rate in light-emitting devices using different materials like $\left(\mathrm{HfO}_{2}, \mathrm{MgF}_{2}\right.$, epoxy, and air) as external medium. Many devices based on light emitting thin films have difficulties when coupling the light into air, because of problemswith light guiding. Using epoxy can enhance the coupling of light from the layer into the air, below the critical angle; the epoxy diffracts the internal light with a large solid angle into the escape light cone. More than 1.6-fold PL extraction efficiency enhancement was observed from epoxy/GaN/sapphire compared to the cavity without epoxy. 


\section{Acknowledgment}

This work was supported by the UniversitiSains Malaysia through a short term grant 304/PFIZIK/6312076, is greatly appreciated.

\section{References}

[1] N. Holonyak, Is the light emitting diode (LED) an ultimate lamp? Amer. J. Phys., 68 (2000) 864-866.

[2] M. Rattier, H. Benisty, R.P. Stanley, J.-F. Carlin, R. Houdré, U. Oesterle, C.J.M. Smith, C. Weisbuch, T. F. Krauss, Illumination With Solid State Lighting Technology, IEEE Journal of Selected Topics in Quantum Electronics, 8 (2), March/April (2002) 310-320

[3] H. An-He, Z. Yong, Z. Xue-Hui, C. Xian-Wen, F. Guang-Han, H. Miao, Improved light extraction of GaN-based light-emitting diodes with surface-textured indium tin oxide electrodes by nickel nanoparticle mask dry-etching, Chinese Phys. B 19 (6) (2010) 68101068101.

[4] P.A. Porta,M. Harries, and H.D. Summers, Surface plasmon mediated emission in resonantcavity light-emitting diodes ” Appl. Phys. Lett, 89, ( 2006)121120-1 -121120-3

[5] M. Boroditsky, R. Vrijen, T.F. Krauss, R. Coccioli, R. Bhat, E. Yablonovitch, Spontaneous Emission Extraction and Purcell Enhancement from Thin Film 2-D Photonic Crystals, J. Lightwave Technology, 17 (11) (1999) 2096-2112.

[6] B.J. Scholz, J. Frischeisen, A. Jaeger, D.S. Setz, T.C.G. Reusch, W. Brutting, Extraction of surface plasmons in organic light-emitting diodes viahigh-index coupling, Optics Express 20(S2) (2012) A205-A212.

[7] D. Ochoa, R. Houdré, M. Ilegems, C. Hanke, B. Borchert, Microcavity light emitting diodes as efficient planar light emitters for telecommunication applications, C. R. Physique 3 (2002) 3-14. 
[8] L. Coldren, S. Corzine, Diode Lasers and Photonic Integrated Circuits, JohnWiley\& Sons, 1995.

[9] K. Gillessenand, W. Schrairer, Light Emitting Diodes: an Introduction, Prentice-Hall International Series in Optoelectronics, 1987.

[10] N.M. Ahmed, M.R. Hashim, Z. Hassan, Large enhancement of GaN-UV light emission using silver mirror resonator, Phys. Stat. Sol. C, 3(6) (2006) 2022-2025.

[11] N.M. Ahmed, Z. Sauli, U. Hashim, Z.A.Z. Jamal, Nano-silver microcavity enhanced UV GaN light emitter” Int. J. Nanomanufacturing, Vol.4, No.1/2/3/4, (2009) 26 - 33.

[12] L. K. Wu, W. Z. Shen, Resonant-Cavity-Enhanced Far-Infrared Upconversion Imaging Devices, IEEE J. Quant. Eelect. 43(5) (2007) 411-418.

[13] A. Penzkofer, W. Holzer, H. Tillmann, H.H. Horhold, Leaky-mode emission of luminescent thin films on transparent substrates, Optics Communications 229 (2004) 279-290. 